\title{
Results of the round robin calibration of reference solar cells within the PhotoClass project
}

\author{
Ingo Kröger ${ }^{1, *}$, Dirk Friedrich ${ }^{1}$, Stefan Winter $^{1}$, Elena Salis ${ }^{\mathrm{a}, 2}$, Harald Müllejans ${ }^{\mathrm{a}, 2}$, Diego Pavanello ${ }^{\mathrm{a}, 2}$, \\ Jochen Hohl-Ebinger ${ }^{3}$, Karsten Bothe ${ }^{4}$, David Hinken ${ }^{4}$, Sebastian Dittmann ${ }^{5}$, Gabi Friesen ${ }^{5}$, Martin Bliss ${ }^{6}$, \\ Tom Betts ${ }^{6}$, Ralph Gottschalg ${ }^{\mathrm{b}, \mathrm{c}, 6}$, Lorentz Rimmelspacher ${ }^{7}$, Johannes Stang ${ }^{7}$, Werner Herrmann ${ }^{7}$, and \\ Jimmy Dubard ${ }^{8}$ \\ ${ }^{1}$ Physikalisch-Technische Bundesanstalt, Bundesallee 100, 38116 Braunschweig, Germany \\ 2 European Commission, Joint Research Centre, Directorate C - Energy, Transport and Climate, Energy Efficiency and \\ Renewables Unit, European Solar Test Installation (ESTI), Via Fermi 2749, 21027 Ispra, Italy \\ ${ }^{3}$ Fraunhofer Institute for Solar Energy Systems, Heidenhofstr. 2, 79110 Freiburg, Germany \\ ${ }^{4}$ Institute for Solar Energy Research Hameln (ISFH), Am Ohrberg 1, 31860 Emmerthal, Germany \\ ${ }^{5}$ University of Applied Sciences and Arts of Southern Switzerland - Institute for Applied Sustainability to the Built \\ Environment, (SUPSI-ISAAC), Campus Trevano, 6952 Canobbio, Switzerland \\ ${ }^{6}$ Centre for Renewable Energy Systems Technology (CREST), Department of Electronic and Electrical Engineering, \\ Loughborough University, Loughborough, Leicestershire LE11 3TU, UK \\ 7 TÜV Rheinland Energie und Umwelt GmbH, 51101 Cologne, Germany \\ ${ }^{8}$ Laboratoire national de métrologie et d'essais (LNE), 29 ave Roger Hennequin, 78197 Trappes, France
}

Received: 15 May 2018 / Accepted: 6 September 2018

\begin{abstract}
An intercomparison of terrestrial photovoltaic (PV) calibrations was performed among a number of European calibration and testing laboratories that participated in the European Metrology Research Program (EMRP) project "PhotoClass". The purpose of this intercomparison was to evaluate the comparability of calibration and testing services within the stated uncertainties of the individual laboratories. The calibration objects were two world photovoltaic scale (WPVS)-type reference solar cells, one made from crystalline silicon and one made from GaAs. The calibration value (CV) was the short circuit current under standard test conditions $\left(I_{\mathrm{STC}}\right)$. In conclusion, it was found that the CVs are all consistent within the stated uncertainties. This result strengthens the reliance in the calibration chain and in the PV calibration infrastructure in Europe.
\end{abstract}

Keywords: round robin / reference solar cell / calibration / standard test conditions

\section{Introduction}

Traceable calibration and testing of photovoltaic (PV) devices is the very basis of assuring the worldwide comparability of the electrical parameters of PV products such as the efficiency and the nominal power. This is

\footnotetext{
* Corresponding author: ingo.kroeger@ptb.de

a The views expressed are purely those of the authors and may not in any circumstances be regarded as stating an official position of the European Commission.

b Present address: Fraunhofer Center for Silicon Photovoltaics (CSP), Organisational Unit of the FhG-IMWS, Otto-EissfeldtStrasse 12, 06120 Halle, Germany.

${ }^{\mathrm{c}}$ Present address: Chair for Photovoltaic Energy Systems Faculty of Electrical Engineering, Manufacturing and Economic Engineering (EMW), Hochschule Anhalt, Bernburger Str. 57, 06366 Köthen, Germany.
}

essential for strengthening confidence in PV technology as a reliable source of energy. The calibration chain links the industrial production via calibration and testing laboratories to national metrology institutes (NMI) and hence to the SI units. The most crucial measure for PV traceability is the short-circuit current under standard test conditions $\left(I_{\mathrm{STC}}\right)$ since it correlates directly to the irradiance conditions defined in the IEC 60904-3 standard [1]. Various methods have been developed to calibrate PV devices. The most common are the use of pulsed or steady-state solar simulators [2-4], natural sunlight $[3,5]$ and spectral methods such as the differential spectral responsivity (DSR) method [6-8]. In principle, the calibration value (CV) of the PV device should be independent from the method if traceability is assured and if all necessary corrections are applied. However, every method, every correction procedure and each individual facility lead to different measurement uncertainties, which have to be 


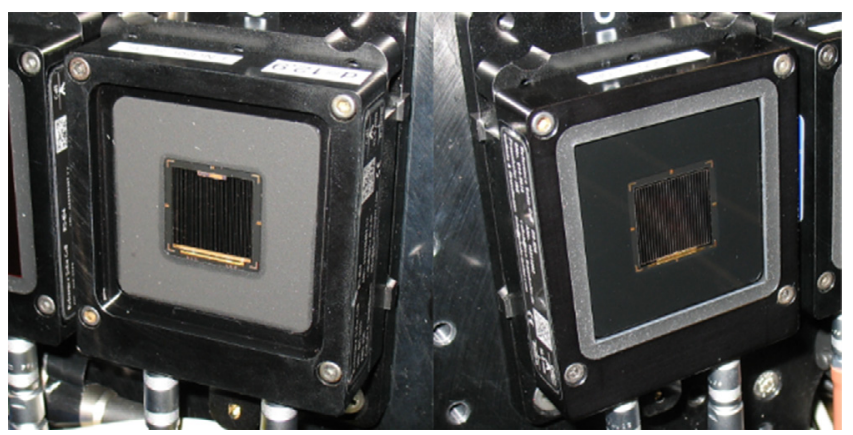

Fig. 1. Picture of the calibration objects ENG55-S-05 (left) and ENG55-S-08 (right).

identified and quantified in order to assign the correct measurement uncertainty to the $\mathrm{CV}$ of the PV device under test (DUT). In order to validate calibration facilities, intercomparison campaigns are performed to compare CVs of a common unknown set of PV devices [9-13].

Here, we show the results of such an intercomparison that was conducted within the European Metrology Research Programme (EMRP) project "PhotoClass". Within "PhotoClass" project the participating calibration and testing laboratories upgraded their facilities to perform energy-rating related measurements. The intention of this intercomparison was the final assessment of these facilities in regard to calibration at standard test conditions (STC), to be used then as reference point for the energy-rating measurements.

\section{Calibration objects}

The calibration objects were two reference solar cells in WPVS [14] design labeled ENG55-S-05 and ENG55-S-08 (see Fig. 1). The ENG55-S-05 is made from a GaAs solar cell without any front glass, the ENG55-S-08 is made from a crystalline n-type silicon solar cell with glass cover encapsulation. In a previous study, reference devices identical in construction were investigated regarding longterm stability of the short-circuit current under enhanced UV-exposure and proved to be stable $<0.10 \%$ [15]. For crosschecking the long-term stability of the reference cells used here, both calibration objects were calibrated at the beginning and at the end of the round robin by the coordinator laboratory $\# 1$. The CV for ENG55-S-08 decreased by $0.16 \%$ and for ENG55-S-05 by $0.20 \%$. However, since both observed changes are within the stated reproducibility of $0.20 \%$ of the calibration facility of laboratory $\# 1$, the reference solar cells can be considered to be stable.

\section{Instrumentation of participating laboratories}

In this section, the calibration facilities and calibration procedure of the participating laboratories will be outlined.

\subsection{Laboratory\#1}

The calibration facility of laboratory $\# 1$ is a DSR facility. The monochromatic irradiance is generated by a tunable laser system and a monochromator system followed by an imaging optics generating a uniform illumination of the DUT. The DUT is simultaneously illuminated with white bias light generating bias irradiance levels from 0 to $1100 \mathrm{~W} / \mathrm{m}^{2}$. The absolute DSR of the DUT is measured at different bias irradiance levels in order to determine the non-linearity of the DSR. The spectral responsivity (SR) as well as the $I_{\text {STC }}$ is then derived mathematically from the DSR measurements using the tabulated AM1.5G spectral irradiance from [1]. The reference detector is a calibrated photodiode traceable to SI via the cryogenic radiometer at Physikalisch-Technische Bundesanstalt (PTB) in Germany.

\subsection{Laboratory\#2}

The calibration facility of laboratory $\# 2$ is a steady-state solar simulator (Class AAA). The two reference cells (DUTs) were calibrated against another c-Si PV reference cell, which is traceable to the world radiometric reference (WRR) and SI irradiance scales establishing the world photovoltaic scale (WPVS). The reference cell and each DUT were consecutively placed into the exact same position on the test plane, thereby eliminating contributions from spatial non-uniformity of the simulated sunlight. The short-circuit current was measured with a dedicated transimpedance amplifier. The spectral mismatch (IEC 60904-7) [16] was corrected based on the spectral irradiance of the solar simulator as measured with a spectroradiometer traceable to SI irradiance scale via a standard lamp calibrated at the National Physical Laboratory (NPL) in the United Kingdom. The SR of the two DUTs was measured according to IEC 60904-8 [17] with a dedicated set-up against a c-Si reference cell calibrated in SR at PTB.

\subsection{Laboratory\#3}

Laboratory $\# 3$ uses an indoor calibration procedure with a Pasan $3 b$ pulsed sun simulator (Class AAA). The primary calibrated reference with traceability to PTB and the DUT are mounted close to the optical axis of the simulator. Both devices are stabilized to $(25 \pm 0.5)^{\circ} \mathrm{C}$ using an active temperature control system. The spectral response of the DUT is measured using the pulsed sun simulator with a set of bandpass filters. Short-circuit current measurements at $(1000 \pm 3) \mathrm{W} / \mathrm{m}^{2}$ are performed close to the zero-crossing over $9 \mathrm{~ms}$ of the simulator's plateau. The spectral output of the sun simulator is measured simultaneously using a calibrated CCD spectrometer. Irradiance and mismatch corrections are applied to determine the short-circuit current at STC conditions.

\subsection{Laboratory\#4}

The calibration procedure in this laboratory is based on a steady-state sun-simulator with a spectral mismatch correction to determine the IV-curve under STC. The DUT is kept at $25^{\circ} \mathrm{C}$ during the measurements (SR and IVmeasurement). The spectral response measurement is 
realized with a filter-based monochromator setup. Traceability is realized with reference cells/diodes calibrated at PTB.

\subsection{Laboratory\#5}

Laboratory \#5 uses a Pasan 3b pulsed light solar simulator to measure the IV-curve of PV devices. The reference and DUT are stabilized at $25^{\circ} \mathrm{C}$ using room temperature control. The spectral output of the solar simulator is measured in situ using a calibrated CCD spectroradiometer with traceability of the standard lamp to the National Institute of Standards and Technology (NIST) in the United States of America. The SR of samples is measured using a filter-based set-up with the DUT controlled at $25^{\circ} \mathrm{C}$. The internal reference photodiode is calibrated using a reference cell with traceability to the European Solar Test Installation at Joint Research Center of the European Commission (JRC-ESTI). A mismatch correction is applied to determine the IV-curve at STC conditions.

\subsection{Laboratory\#6}

The calibration facility of laboratory $\# 6$ is a DSR and IV facility. At the DSR facility, the monochromatic irradiance is generated by a xenon/halogen lamp system and a monochromator in combination with optical filters followed by an imaging optics generating a uniform illumination on the DUT. The DUT is simultaneously illuminated with white bias light with irradiances from 0 to $1100 \mathrm{~W} / \mathrm{m}^{2}$. The relative DSR of the DUT is measured at different bias irradiancelevels in order to determine the non-linearity of the DSR. The relative (non-differential) SR is derived mathematically from the DSR data using the tabulated AM1.5G spectral irradiance. The relative SR curve of the DUT is used to calculate the spectral mismatch correction factor which is then used at the IV facility to determine $I_{\mathrm{STC}}$. The IV facility comprises a steady-state xenon/halogen lamp system (classified to AAA) with a built-in spectroradiometer. The determined $I_{\mathrm{STC}}$-value is used to scale the relative differential and relative non-differential SR curves to absolute units. The references of both facilities are WPVS-type solar cells calibrated at PTB.

\subsection{Laboratory\#7}

The indoor calibration procedure is based on a side-by-side comparison with a primary calibrated WPVS-type c-Si reference cell. The measurements are performed with a Pasan 3b pulsed solar simulator (Class AAA) at an irradiation level of $1000 \mathrm{~W} / \mathrm{m}^{2}$. Diffuse light in the test area is avoided by use of baffles in the beam of light. Both cells are installed close to the optical axis and connected to precision shunt resistors, which operate the cells near short-circuit conditions (voltage drop 20-30 mV). During the flash pulse the signals of the DUT and the reference device are recorded simultaneously with a transient recorder and the average signals during the $8 \mathrm{~ms}$ plateau are calculated. Finally, the resulting value of the DUT is spectrally and irradiance corrected by referencing the data of the reference device.

\subsection{Laboratory\#8}

The calibration facility of laboratory $\# 8$ is a pulsed solar simulator Pasan 3C (class AAA). Measurement of the cells $I_{\mathrm{SC}}$ is determined from the $\mathrm{I}-\mathrm{V}$ curve that is measured on a time scale of $10 \mathrm{~ms}$ and at an ambient temperature of $(25 \pm 1)^{\circ} \mathrm{C}$. The irradiance at $1000 \mathrm{~W} / \mathrm{m}^{2}$ is monitored by a reference cell that is placed in the same plane as the DUT. The Si reference cell is calibrated by Fraunhofer-Institut für Solare Energiesysteme ISE.

\subsection{Laboratory\#9}

The calibration facility of laboratory $\# 9$ is a DSR facility. The monochromatic irradiance is generated by a xenon/ halogen lamp system and a double-monochromator system followed by an imaging optics generating a uniform illumination of the DUT. The DUT is simultaneously illuminated with white light bias generating Bias irradiance levels from 0 to $1100 \mathrm{~W} / \mathrm{m}^{2}$. The absolute DSR of the DUT is measured at different Bias irradiance level in order to determine the non-linearity of the DSR. The SR as well as the $I_{\mathrm{STC}}$ is derived mathematically from the DSR measurements using the tabulated AM1.5G spectral irradiance from [1]. The reference is a calibrated photodiode traceable to SI via the cryogenic radiometer at PTB.

\section{Measurement results}

The measurand for this intercomparison is the $\mathrm{CV}$ of the short-circuit current under STC, which are $E_{\mathrm{STC}}=1000$ $\mathrm{W} / \mathrm{m}^{2}$ total irradiance, $25^{\circ} \mathrm{C}$ solar cell temperature and AM1.5G reference solar spectral irradiance distribution defined in IEC 60904-3 [1]. Additionally, a comparison of the measurement results for the relative SR $s_{\text {rel }}(\lambda)$ is shown. These SRs have been used for spectral mismatch correction $[16,18]$ of the short-circuit current as measured by all methods other than DSR. The spectral mismatch correction must be applied to the CV of a solar cell, if either the spectral irradiance distribution of the light source $E_{\lambda}(\lambda)$ differs from the AM1.5G spectral irradiance $E_{\lambda \text {,ref }}(\lambda)$ or the relative SR of the DUT $s_{\mathrm{DUT}}(\lambda)$ differs from the relative SR of the reference $s_{\text {rel }}(\lambda)$. This spectral mismatch correction (SMM) factor is calculated by the following formula:

$$
S M M=\frac{\int_{\lambda} E_{\lambda, \mathrm{ref}}(\lambda) \cdot s_{\mathrm{ref}}(\lambda) d \lambda}{\int_{\lambda} E_{\lambda}(\lambda) \cdot s_{\mathrm{ref}}(\lambda) d \lambda} \cdot \frac{\int_{\lambda} E_{\lambda}(\lambda) \cdot s_{\mathrm{DUT}}(\lambda) d \lambda}{\int_{\lambda} E_{\lambda, \mathrm{ref}}(\lambda) \cdot s_{\mathrm{DUT}}(\lambda) d \lambda}
$$

The output of this spectral correction is the CV assigned to the DUT by each laboratory. This is valuable information since the spectral mismatch correction is a dominant uncertainty contribution if the relative SR of the reference device and the DUT differ significantly and if the spectrum used for measurement is not well matched to the AM1.5 reference spectrum [18]. 


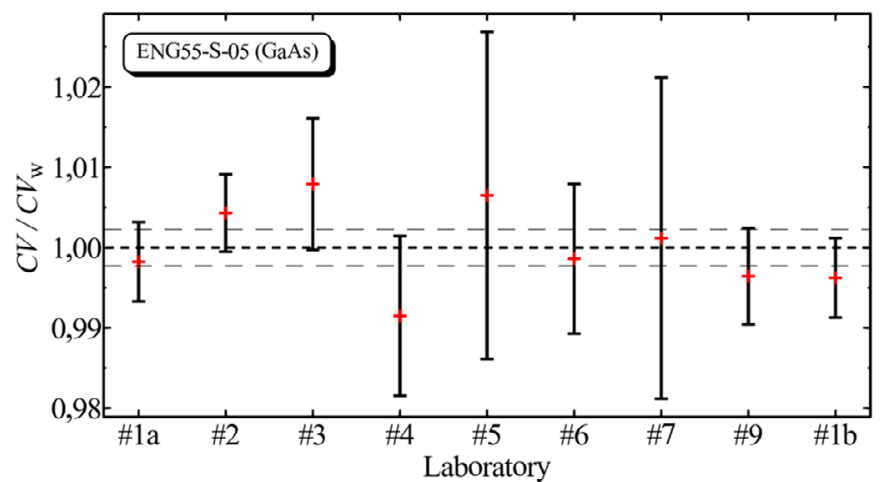

Fig. 2. Comparison of the calibration values for the GaAs reference solar cell.

\subsection{Data treatment}

The CVs $\mathrm{CV}_{i}$ and the assigned expanded uncertainties $U$ $\left(\mathrm{CV}_{i}\right)$ were submitted by the participating laboratories to the coordinator laboratory $\# 1$ for data treatment. From these CVs the weighted mean and the expanded uncertainty of the weighted mean were calculated $[19,20]$. Since the coordinator laboratory contributes two measurements $(\# 1 \mathrm{a}, \# 1 \mathrm{~b})$ to the intercomparison, the weights of both measurements were multiplied by a factor of 0.5 :

$$
\begin{gathered}
C V_{w}=\left(\sum_{i=1}^{n} C V_{i} w_{i}\right) / \sum_{i}^{n} w_{i}, \\
\text { with } w_{i}=U\left(C V_{i}\right)^{-2}, \\
U\left(C V_{w}\right)=\frac{1}{\sqrt{\sum_{i=1}^{n} \frac{1}{U\left(C V_{i}\right)^{2}}}} .
\end{gathered}
$$

Then the individual measured CVs were normalized to the weighted mean.

To check the consistency of the CVs, the individual $E_{n}$ numbers according to ISO/IEC 17043 [21] were calculated following::

$$
E_{n, i}=\frac{C V_{i}-C V_{w}}{\sqrt{U\left(C V_{i}\right)^{2}+U\left(C V_{w}\right)^{2}}} .
$$

If $\left|E_{n}\right| \leq 1$ the measurements can be considered as consistent within their stated uncertainties.

\subsection{Short-circuit current $\left(I_{\mathrm{STC}}\right)$}

In Figures 2 and 3 the measured values normalized to the weighted mean are shown. The error bars denote the expanded uncertainty of the individual CVs. The longdashed lines denote the expanded uncertainty of the weighted mean according to equation (4). Please note that the stated measurement uncertainties of laboratory $\# 1$, laboratory $\# 2$ and laboratory $\# 9$ are significantly lower than those of the other laboratories. Hence, the value of the weighted mean is dominated by these three laboratories.

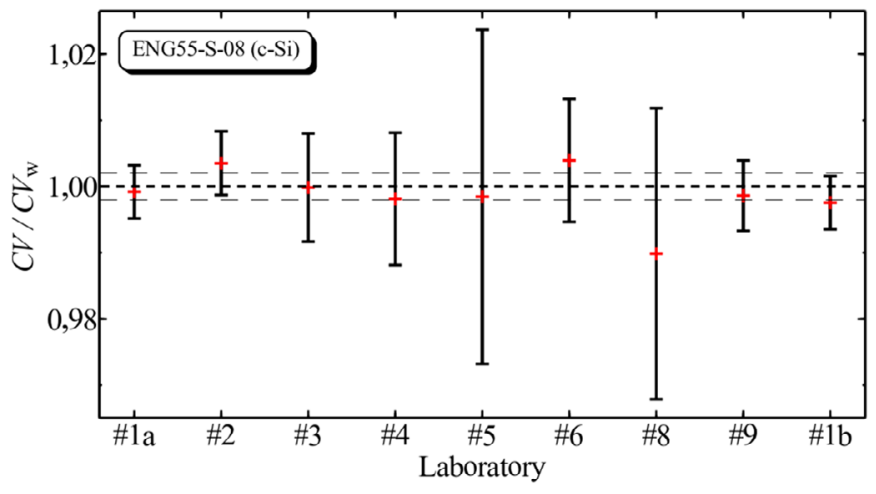

Fig. 3. Comparison of the calibration values for the c-Si reference solar cell.
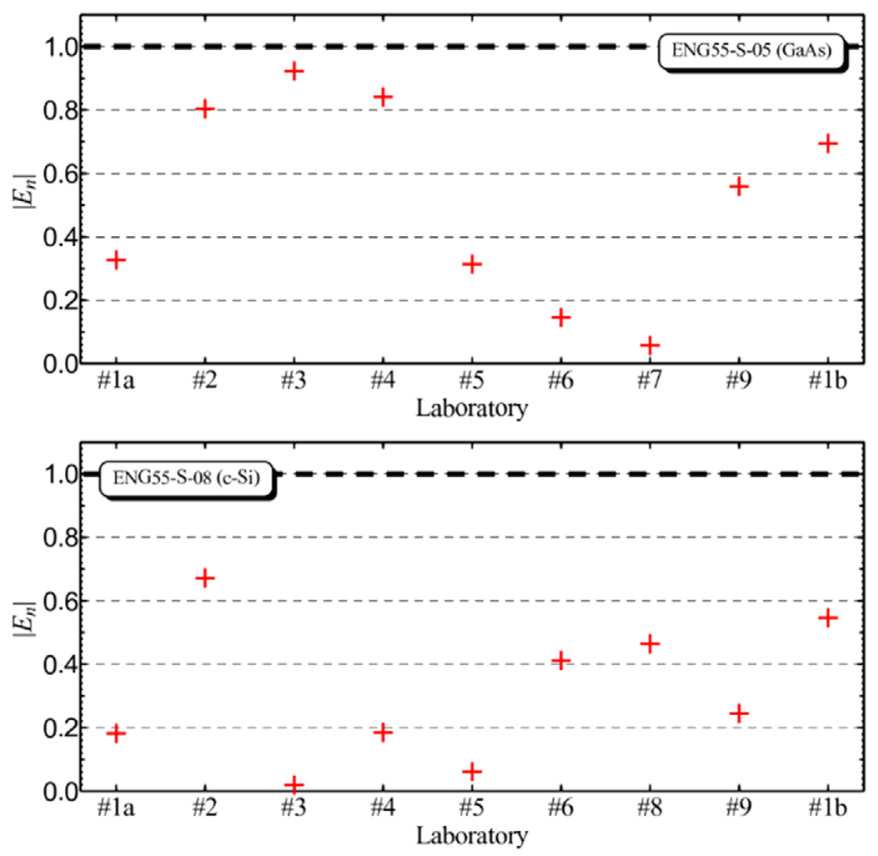

Fig. 4. Absolute $E_{n}$ numbers of the individual calibration values with respect to the weighted mean according to ISO17043.

The $1.04 \%$ standard deviation of the CVs of GaAs is slightly higher compared to the $0.81 \%$ of the c-Si reference solar cell. This most likely results from higher intrinsic measurement uncertainties related to a higher impact of the spectral mismatch correction on the CV, when methods other than DSR are used.

The $\left|E_{n}\right|$ values according to equation (5) are shown in Figure 4 . All $E_{n}$ values are smaller than 1 and hence these calibrations can be considered consistent within their stated uncertainties. Please note that the high $E_{n}$ values of laboratory $\# 1, \# 2$ and $\# 9$ result mainly from their low stated uncertainties. In accordance to the Bureau International des Poids et Mesures (BIPM) recommendations for Consultative Committee for Photometry and Radiometry (CCPR) key comparisons, the data analysis can be performed introducing a cutoff value for the uncertainty in order to reduce the effect of $\mathrm{CVs}$ of laboratories with the lowest uncertainties on the weighted mean CV. This 

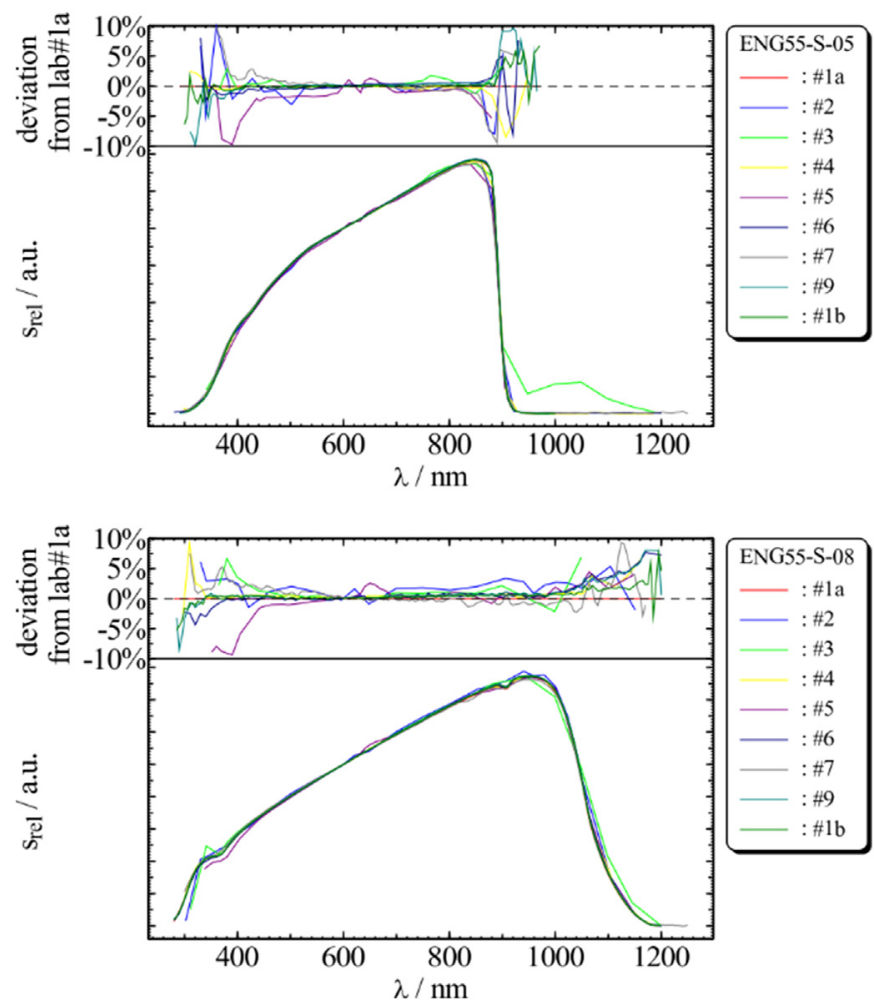

Fig. 5. The lower graphs show the measured SRs $s(\lambda)$ of the different laboratories normalized to $s(600 \mathrm{~nm})$. The upper graphs show the relative deviation to measurement of the pilot laboratory $\# 1$.

analysis was additionally done. However, this procedure only had a negligible effect on the intercomparison results discussed above.

\subsection{Relative SR}

In context to the $\mathrm{CVS}$ of the $I_{\mathrm{STC}}$ of the two reference devices, most of the participating laboratories additionally submitted the relative SR. A comparison of these measurements is of additional value since the $\mathrm{SR}$ measurement is together with the spectral irradiance measurement an input quantity for spectral mismatch calculations. Measurement uncertainties of the SR measurement propagate into measurement uncertainties of the spectral mismatch correction factor [18] and hence of the CV of the $I_{\mathrm{STC}}$.

The lower graphs of Figure 5 show the measured SRs $s(\lambda)$ of the different laboratories normalized to $s(600 \mathrm{~nm})$. The upper graph shows the deviation relative to the measurement of coordinator laboratory\#1, which was agreed to represent the reference measurement. Unfortunately, no $E_{n}$ numbers could be derived for these measurements since some submitted data lacked stated uncertainties for the SR measurement.

Generally, the agreement of the SR measurements can be considered to be good and sufficient for SMM. Exemplary spectral mismatch correction factor calculations were performed using the formula (1) [15]. In this case, $E_{\lambda, \text { ref }}(\lambda)$ is the AM1.5G spectral irradiance, for $E_{\lambda}(\lambda)$ a typical spectral irradiance of an AAA pulsed solar simulator was taken, for $s_{\text {ref }}(\lambda)$ the relative spectral irradiance of the ENG55-S-08 device and for $s_{\mathrm{DUT}}(\lambda)$ the relative spectral irradiance of the ENG55-S-05 device was taken for the individual laboratory. The maximum difference of the resulting SMM factors was found to be $0.21 \%$, which can be considered to be the impact of an SR measurement uncertainty via the spectral mismatch correction factor on the CV for this particular case. This magnitude agrees well with the observed $1.04 \%$ standard deviation of the CVs of the GaAs DUT where spectral mismatch correction is of major importance compared to the $0.81 \%$ of the c-Si DUT where spectral mismatch correction is only of minor importance (if a c-Si reference was used).

\section{Conclusion}

A round-robin intercomparison on the calibration of the short-circuit current under STC of a c-Si and a GaAs reference solar cell was performed. Among the participating calibration laboratories different methods and instrumentation were applied, mainly pulsed solar simulators, steady-state solar simulators and the DSR method. Each laboratory evaluated the measurement uncertainty of the $\mathrm{CV}$ according to its internal procedures. According to the derived $E_{n}$ values all CVs can be considered consistent within the stated uncertainties. Hence, international equivalence of traceable calibration of PV devices can be stated. This result strengthens the reliance in the calibration chain and in the PV calibration infrastructure in Europe.

The research work leading to this article was carried out within the EMRP ENG55 project "Towards an energy-based parameter for photovoltaic classification" (PhotoClass). The EMRP is jointly funded by the EMRP participating countries within EURAMET and the European Union.

\section{References}

1. IEC60904-3:2008, Photovoltaic Devices - Part 3: Measurement Principles for Terrestrial Photovoltaic (PV) Solar Devices with Reference Spectral Irradiance Data (International Electrotechnical Commission, Geneva, 2008)

2. Y. Hishikawa, S. Igari, H. Kato, I. Tsuda, Calibration and measurement of solar cells and modules by the solar simulator method in Japan, in Proceedings of the 3rd World Conference onPhotovoltaic Energy Conversion, 2003, Osaka, Japan (2003)

3. H. Müllejans, W. Zaaiman, F. Merli, E. Dunlop, H. Ossenbrink, Comparison of traceable calibration methods for primary photovoltaic reference cells, Prog. Photovolt.: Res. Appl. 13, 661 (2005)

4. S. Ahn, S.Y.J. Ahn, D. Lee, S. Winter, S. Igari, K. Yoon, Establishment of a primary reference solar cell calibration technique in Korea: methods, results and comparison with WPVS qualified laboratories, Metrologia 51, 139 (2014) 
5. H. Müllejans, W. Zaaiman, E. Dunlop, H. Ossenbrink, Calibration of photovoltaic reference cells by the global sunlight method, Metrologia 42, 360 (2005)

6. S. Winter, T. Wittchen, J. Metzdorf, Primary reference cell calibration at the PTB based on an improved DSR facility, in Sixteenth European Photovoltaic Solar Energy Conference: Proceedings, Glasgow (2000)

7. J. Metzdorf, Calibration of solar cells. 1: The differential spectral responsivity method, Appl. Opt. 26, 1701 (1987)

8. S. Winter, T. Fey, I. Kröger, D. Friedrich, B.O.S.P.K. Ladner, F. Witt, Design, realization and uncertainty analysis of a laser-based primary calibration facility for solar cells at PTB, Measurement 51, 457 (2014)

9. E. Salis, D. Pavanello, M. Field, U. Kräling, F. Neuberger, K. Kiefer, C. Osterwald, S. Rummel, D. Levi, Y. Hishikawa, K. Yamago, H. Ohshima, M. Yoshita, H. Müllejans, Improvements in world-wide intercomparison of PV module calibration, Solar Energy 155, 1451 (2017)

10. Y. Hishikawa, H. Liu, H.-H. Hsieh, T. Inoue, K.-S. Kim, C. Limsakul, S.-W. Kim, R. Ninae, K. Morita, Round-robin measurement intercomparison of c-Si PV modules among Asian testing laboratories, Prog. Photovolt. Bd. 21, 1181 (2013)

11. D. Dirnberger, U. Kräling, H. Müllejans, E. Salis, K. Emery, Y. Hishikawa, K. Kiefer, Progress in photovoltaic module calibration: results of a worldwide intercomparison between four reference laboratories, Meas. Sci. Technol. 25, 105005 (2014)

12. K. Emery, The results of the Forst World Photovoltaic Scale Recalibration, NREL Technical Report, 2000
13. S. Winter, J. Metzdorf, K. Emery, F. Fabero, Y. Hishikawa, B. Hund, H. Müllejans, W. Warta, The results of the second world photovoltaic scale recalibration, in 31st IEEE Photovoltaic Specialists Conference and Exhibition, Orlando, USA (2005)

14. C. Osterwald, S. Anevsky, K. Bücher, A. Barua, P. Chaudhuri, J. Dubard, K. Emery, B. Hansen, D. King, J. Metzdorf, F. Nagamine, R. Shimokawa, Y. Wang, T. Wittchen, W. Zaaiman, A. Zastrow, J. Zhang, The world photovoltaic scale: an international reference cell calibration program, Prog. Photovolat.: Res. Appl. 7, 287 (1999)

15. I. Kröger, J. Hohl-Ebinger, S. Brachmann, S. Winter, Investigation of UV-Induced degradation of different types of WPVS reference solar cells, in 32nd European Photovoltaic Solar Energy Conference and Exhibition, Munich (2016)

16. IEC60904-7:2009, Photovoltaic Devices - Part 7: Computation of the Spectral Mismatch Correction for Measurements of Photovoltaic Devices (International Electrotechnical Commission, Geneva, 2009)

17. 60904-8:2014, Photovoltaic Devices - Part 8: Measurement of Spectral Responsivity of a Photovoltaic (PV) Device (International Electrotechnical Commission, Geneva, 2014)

18. J. Hohl-Ebinger, W. Warta, Uncertainty of the spectral mismatch correction factor in STC measurements on photovoltaic devices, Prog. Photovol. 19, 573 (2011)

19. M.G. Cox, The evaluation of key comparison data, Metrologia 39, $589(2002)$

20. H. Müllejans, W.Zaaiman,E.Dunlop, Reduction of uncertainties for photovoltaic reference solar cells, Metrologia 52, 646 (2015)

21. ISO/IEC17043, Conformity Assessment - General Requirements for Proficiency Testing (2010)

Cite this article as: Ingo Kröger, Dirk Friedrich, Stefan Winter, Elena Salis, Harald Müllejans, Diego Pavanello, Jochen HohlEbinger, Karsten Bothe, David Hinken, Sebastian Dittmann, Gabi Friesen, Martin Bliss, Tom Betts, Ralph Gottschalg, Lorentz Rimmelspacher, Johannes Stang, Werner Herrmann, Jimmy Dubard, Results of the round robin calibration of reference solar cells within the PhotoClass project, Int. J. Metrol. Qual. Eng. 9, 8 (2018) 